\title{
Defining bacterial species in the genomic era: insights from the genus Acinetobacter
}

\author{
Jacqueline Z-M Chan ${ }^{\dagger}$, Mihail R Halachev ${ }^{\dagger}$, Nicholas J Loman, Chrystala Constantinidou and Mark J Pallen ${ }^{*}$
}

\begin{abstract}
Background: Microbial taxonomy remains a conservative discipline, relying on phenotypic information derived from growth in pure culture and techniques that are time-consuming and difficult to standardize, particularly when compared to the ease of modern high-throughput genome sequencing. Here, drawing on the genus Acinetobacter as a test case, we examine whether bacterial taxonomy could abandon phenotypic approaches and DNA-DNA hybridization and, instead, rely exclusively on analyses of genome sequence data.

Results: In pursuit of this goal, we generated a set of thirteen new draft genome sequences, representing ten species, combined them with other publically available genome sequences and analyzed these 38 strains belonging to the genus. We found that analyses based on $16 \mathrm{~S}$ rRNA gene sequences were not capable of delineating accepted species. However, a core genome phylogenetic tree proved consistent with the currently accepted taxonomy of the genus, while also identifying three misclassifications of strains in collections or databases. Among rapid distance-based methods, we found average-nucleotide identity (ANI) analyses delivered results consistent with traditional and phylogenetic classifications, whereas gene content based approaches appear to be too strongly influenced by the effects of horizontal gene transfer to agree with previously accepted species.

Conclusion: We believe a combination of core genome phylogenetic analysis and ANI provides an appropriate method for bacterial species delineation, whereby bacterial species are defined as monophyletic groups of isolates with genomes that exhibit at least 95\% pair-wise ANI. The proposed method is backwards compatible; it provides a scalable and uniform approach that works for both culturable and non-culturable species; is faster and cheaper than traditional taxonomic methods; is easily replicable and transferable among research institutions; and lastly, falls in line with Darwin's vision of classification becoming, as far as is possible, genealogical.
\end{abstract}

Keywords: Genome-based taxonomy, Bacteria, Sequence-based analysis, Whole-genome data

\section{Background}

In the early eighteenth century, Linnaeus provided the first workable hierarchical classification of species, based on the clustering of organisms according to their phenotypic characteristics [1]. In The Origin of Species [2], Darwin added phylogeny to taxonomy, while also emphasizing the arbitrary nature of biological species: "I look at the term species as one arbitrarily given for the sake of convenience to a set of individuals resembling each other." The reality and utility of the species concept continues to inform the theory and practice of biology

\footnotetext{
* Correspondence: m.pallen@bham.ac.uk

${ }^{\dagger}$ Equal contributors

Institute of Microbiology and Infection, School of Biosciences, University of Birmingham, Edgbaston B15 2TT, UK
}

\section{() Biomed Central}

and a stable species nomenclature underpins the diagnosis and monitoring of pathogenic microorganisms [3-5].

Traditional taxonomic analyses of plants and animals rely on morphological characteristics. However, this approach cannot easily be applied to unicellular microorganisms. In the latter half of the twentieth century, it became clear that bacteria could be grouped into taxonomic clusters based on stable phenotypic characters (e.g. cellular morphology and composition, growth requirements and other metabolic traits) that could be measured reliably in the laboratory. In the 1960s and 1970s, Sneath and Sokal exploited improved technical and statistical methods to develop a numerical taxonomy, which revealed discrete phenotypic clustering within many bacterial genera [6]. 
Such phenotypic approaches soon faced competition from genotypic approaches, such as DNA base composition (mol\% G+C content) [7] and whole-genome DNADNA hybridization (DDH); the latter remains the gold standard in bacterial taxonomy [8]. Within this framework, Wayne et al. [8] recommended that "a species generally would include strains with approximately $70 \%$ or greater DNA-DNA relatedness". However, few laboratories now perform DNA-DNA hybridization assays as these are onerous and technically demanding when compared to the rapid and easy sequencing of small signature sequences, such as the $16 \mathrm{~S}$ ribosomal RNA gene. This shift has led to an updated species definition: "a prokaryotic species is considered to be a group of strains that are characterized by a certain degree of phenotypic consistency, showing 70\% of DNA-DNA binding and over $97 \%$ of $16 \mathrm{~S}$ ribosomal RNA (rRNA) gene-sequence identity" [9].

Most recently, whole-genome sequencing has delivered new taxonomic metrics-for example, average nucleotide identity (ANI), calculated from pair-wise comparisons of all sequences shared between any two strains. ANI exhibits a strong correlation with DDH values [10], with an ANI value of $\geq 95 \%$ corresponding to the traditional $70 \%$ DDH threshold [10].

Despite the ready availability of genome sequence data, microbial taxonomy remains a conservative discipline. When defining a bacterial species, most modern microbial taxonomists use a polyphasic approach, whereby a bacterial species represents "a monophyletic and genomically coherent cluster of individual organisms that show a high degree of overall similarity with respect to many independent characteristics, and is diagnosable by a discriminative phenotypic property" [11]. Although the polyphasic approach is pragmatic and widely applicable, it has drawbacks. It relies on phenotypic information, which in turn relies on growth, usually in pure culture, in the laboratory, which may not be achievable for many bacterial species [12]. It also relies on techniques that are time-consuming and difficult to standardize, particularly when compared to the ease of modern genome sequencing $[4,13,14]$.

We, like others, are therefore driven to consider whether, in the genomic era, bacterial taxonomy could, and should, abandon phenotypic approaches and rely exclusively on analyses of genome sequence data [4,10,14-18]. However, such an approach brings fresh conceptual and methodological challenges. Several forces shape the evolution of bacterial genomes: the steady accumulation of point mutations or small insertions/deletions (indels), potentially giving rise to a tree-like phylogeny; the influence of homologous recombination in some lineages, obscuring such diversification; and the key role of gene gain/loss, particularly the pervasive influence of horizontal gene transfer, which, if substantial, could obliterate phylogenetic signals. These forces act with different strength on different parts of the genome and on different bacterial lineages. For example, sequences from a single gene such as the 16S rRNA gene have been shown to fail to capture the true genome-wide divergence between two strains [19-21]. Additionally, it may be expected that the various novel sequence-based metrics would be affected differently by different evolutionary forces. This raises potential problems with the consistency of classification (results may or may not be consistent across the metrics) and backwards compatibility (classification may or may not correspond to already named species within a genus). In this work, we wished to explore these issues on a well-characterized and important bacterial genus, Acinetobacter.

The genus Acinetobacter was first proposed by Brisou and Prévot in 1954 [22]; however, it was not until Baumann et al. [23] published their comprehensive study based on nutritional and biochemical properties that this designation became more widely accepted. In 1974 the genus was listed in Bergey's Manual of Systematic Bacteriology with the description of a single species, A. calcoaceticus. To date, there are 27 species described in the genus (http://www.bacterio.cict.fr/a/acinetobacter. html). To fall within genus Acinetobacter, isolates must be Gram-negative, strictly aerobic, non-fermenting, nonfastidious, non-motile, catalase-positive, oxidase-negative and have a DNA G+C content of 38-47\% [24]. Some isolates within the genus are naturally competent resulting in intra-species recombination [25-27]. Environmental isolates, such as A. calcoaceticus PHEA-2 and Acinetobacter oleivorans DR1, have attracted interest because they are able to metabolize a diverse range of compounds [28-30]. However, most research on the genus has focused on clinical isolates, particularly from the species $A$. baumannii. This species has shown an astonishing ability to acquire antibiotic resistance genes and some strains are now close to being untreatable [31,32]. Worryingly, the incidence of serious infections caused by other Acinetobacter species is also increasing [33]. Genotypic approaches have suggested that $A$. baumannii forms a complex-the A. baumannii/calcoaceticus or ACB complex-with three other species $A$. calcoaceticus, A. nosocomialis and A. pittii. However, it remains very difficult, if not impossible, for a conventional reference laboratory to distinguish these species on phenotypic grounds alone [34]. Techniques such as AFLP and amplified 16S rRNA gene restriction analysis (ARDRA) can be used to identify species within the Acinetobacter genus and the ACB complex [35-38]; however, these techniques are too laborious to be carried out in a routine laboratory [24].

Given the general difficulty in defining bacterial species and the ready availability of genome sequence data, 
we sought to evaluate a range of novel genotypic and genome-based metrics for species delineation. In light of discussed obstacles and the on-going public health concern, we believe that genus Acinetobacter provides a timely test case to evaluate the validity and robustness of these sequence-based approaches. In pursuit of this goal, we generated a diverse and informative set of thirteen new draft genome sequences, representing ten species, and we analyzed the whole-genome sequences from a total of 38 strains belonging to the genus.

\section{Results and discussion}

\section{General genome characteristics}

The genomes of thirteen Acinetobacter strains, including seven type strains, were sequenced to draft quality using 454 sequencing (Table 1). The A. bereziniae strain was found to have the largest genome size within the genus ( $5 \mathrm{Mb}$ ), while the strain with the smallest genome $(\sim 2.9 \mathrm{Mb})$ belonged to the species A. parvus, which is known to have a reduced metabolic repertoire compared to other Acinetobacter species [39]. These thirteen genomes were considered alongside twenty-five other publicly available genome sequences from the genus Acinetobacter (see Additional file 1).

\section{A. ursingii DSM 16037 genome characteristics}

The species $A$. ursingii was first described by Nemec et al. in 2001 [40]. We have genome sequenced the type strain DSM 16037, which was isolated from a blood culture taken from an inpatient in Prague, Czech Republic in 1993 [40]. In the genome we identified 3252 goodquality CDSs (minimum length 50 codons of which less than $2 \%$ are stop codons); 270 of these do not have homologs in any of the other 37 Acinetobacter strains in this study. Depth of coverage was generally consistent, apart from two contigs which showed 3.5 times greaterthan-average coverage. Scrutiny of the larger of these two contigs $(9.4 \mathrm{~kb})$ identified CDSs that are predicted to encode plasmid replication and mobilization proteins. This contig also contains homologs of sul1 and usp $A$ genes, which are often associated with $A$. baumannii resistance islands [41].

\section{A. Iwoffii NCTC 5866 genome characteristics}

A. lwoffii was first described by Audureau in 1940 under the name Moraxella lwoffii [22], but was later moved to genus Acinetobacter by Baumann et al. [23]. In 1986, Bouvet and Grimont emended the description of the species to designate strain NCTC 5866 the type strain [42]. We identified 3005 good-quality CDSs in the NCTC 5866 genome, of which 229 do not have homologs in any of the Acinetobacter genomes examined in this study. Investigation of these CDSs revealed two putative prophages, $c a .44 .5$ and $25.6 \mathrm{~kb}$. Interestingly, many of the CDSs found in these two putative prophages are also present in a recently sequenced environmental Acinetobacter strain P8-3-8 (not included in this study) isolated from the intestine of a blue-spotted cornetfish caught in Vietnam [43].

Among the remaining strain-specific CDSs, we identified fourteen that are nearly identical to tra genes found in PHH1107, a low GC content plasmid isolated from pig manure [44]. The tra homologs are distributed on

Table 1 Genome sizes, sequencing statistics, G+C content, number of CDSs in the thirteen sequenced Acinetobacter isolates

\begin{tabular}{|c|c|c|c|c|c|c|c|}
\hline Species & Strain & $\begin{array}{l}\text { Genome } \\
\text { size }(\mathrm{Mb})\end{array}$ & $\begin{array}{c}\text { Peak } \\
\text { coverage }\end{array}$ & No. of contigs & $\mathrm{G}+\mathrm{C}$ content $(\%)$ & $\begin{array}{l}\text { No. of predicted } \\
\text { good quality } \\
\text { CDSs } t\end{array}$ & $\begin{array}{c}\text { GenBank accession } \\
\text { number }\end{array}$ \\
\hline A. parvus & DSM 16617 (T) & 2.88 & $24 x$ & 257 & 41.6 & 2681 & AIEB00000000 \\
\hline A. radioresistens & DSM 6976 (T) & 3.35 & $13 x$ & 354 & 41.4 & 2964 & AIDZ00000000 \\
\hline A. Iwoffii & NCTC $5866(T)$ & 3.35 & $14 x$ & 260 & 43.0 & 3005 & AIEL00000000 \\
\hline A. ursingii & DSM 16037 (T) & 3.57 & $21 x$ & 158 & 40.0 & 3252 & AIEA00000000 \\
\hline A. pittii* & DSM 21653 (T) & 3.75 & $8 x$ & 468 & 38.8 & 3252 & AIEK00000000 \\
\hline A. calcoaceticus & DSM 30006 (T) & 3.89 & $10 x$ & 373 & 38.6 & 3377 & AIEC00000000 \\
\hline A. baumannii & W6976 & 3.91 & $8 x$ & 537 & 39.0 & 3252 & AIEG00000000 \\
\hline A. baumannii & W7282 & 3.95 & $14 x$ & 140 & 39.0 & 3466 & AlEH00000000 \\
\hline A. baumannii & NCTC 7422 & 3.99 & $22 x$ & 179 & 41.3 & 3626 & AIED00000000 \\
\hline A. pittii* & DSM 9306 & 4.03 & $11 x$ & 339 & 38.8 & 3553 & AlEF00000000 \\
\hline A. nosocomialis* & NCTC 8102 & 4.12 & $10 x$ & 283 & 38.7 & 3596 & AIEJ00000000 \\
\hline A. nosocomialis* & NCTC 10304 & 4.16 & $10 x$ & 387 & 39.1 & 3501 & AIEE00000000 \\
\hline A. bereziniae & LMG 1003 (T) & 4.98 & $12 x$ & 392 & 38.1 & 4480 & AIEI00000000 \\
\hline
\end{tabular}

* Species names as proposed by Nemec et al. [39].

† Definition of good quality CDS is length $\geq 50$ codons, of which less than $2 \%$ are stop codons.

$(T)=$ Type strain 
two contigs, one of which has a GC content (37\%) lower than the genome mean (43\%).

\section{A. parvus DSM 16617 genome characteristics}

Strain DSM 16617 is the type strain for A. parvus isolated from the ear of an outpatient from Pribram, Czech Republic in 1996 [45]. We identified 2681 good-quality CDSs in the DSM 16617 genome, 179 of which do not have homologs in any of the remaining 37 genomes. Analysis with Prophinder [46] identified one $39 \mathrm{~kb}$ putative prophage containing phage-related genes homologs to putative phage-related genes found in A. baumannii and A. oleivorans DR1. We identified an 8kb contig with 2.5 times higher than average depth of coverage, which contains homologs to phage related genes.

\section{A. bereziniae LMG 1003 genome characteristics}

Strain LMG 1003 is the type strain for A. bereziniae, a recently named species by Nemec et al., which has been isolated from various human, animal and environmental sources [47]. We identified 4480 good-quality CDSs in the genome, with 1061 strain-specific CDSs (no homologs in the rest of the 37 genomes). This is a considerably higher percentage, 24\%, than in other Acinetobacter strains (see Additional file 1). Many of the strain-specific CDSs form clusters of four or more CDSs, with the largest cluster containing 49 consecutive CDSs, of which 45 are strain-specific. Twenty-one CDSs in this cluster have no significant similarity to proteins in the nonredundant protein database.

Depth of coverage analysis revealed several contigs with higher than average value. One such contig has 5 times greater coverage compared to the rest of the genome, which suggests it is a mobile element. It contains a CDS homologous to the sul1 gene often found in A. baumannii resistance islands [41].

\section{A. radioresistens DSM 6976 genome characteristics}

A. radioresistens strain DSM 6976 was isolated in 1979 from cotton sterilized by $\gamma$-radiation and is the type strain for the species [48]. We identified 2964 goodquality CDSs in the genome, of which 188 do not have homologs in any of the remaining 37 genomes.

A comparison with two previously sequenced A. radioresistens, SK82 and SH164, reveals that the three strains share 2458 CDSs (about $83 \%$ of the average number of CDSs in these three strains), 43 of which were not found in the remaining 35 Acinetobacter genomes. Among these there is a homolog of the metE gene, and two genes involved in the degradation of benzoate, an aromatic compound which is known to support the growth of a number of $A$. radioresistens [49]. Though the three strains are quite similar, we identified 143 CDSs in DSM 6976 which are absent in SK82 and SH164, but do have homologs in other Acinetobacter genomes. Within this group there is a genomic island containing nine genes related to fructose metabolism and a cluster of four CDSs predicted to encode for type IV pilin proteins.

\section{Phylogenetic relationships within genus Acinetobacter}

Stackebrandt and Goebel suggested that bacterial species can be delineated using 16S rRNA gene sequences: according to their criteria, when two aligned sequences exhibit $\geq 97 \%$ identity, the isolates from which they originate are deemed to belong to the same species [50]. However, when we extracted 16S rRNA gene sequences from the Acinetobacter genomes in this study, we found that these criteria gave inconsistent results. For example, the 16S rRNA genes from the type strains of A. baumannii and $A$. radioresistens exhibit $97 \%$ sequence identity, suggesting they should be in the same species. Similarly, sequences from the type strains of $A$. calcoaceticus and $A$. lwoffii show $97.6 \%$ identity, again suggesting they should be classified in the same species. Recent studies by Keswani and Whitman [51] and Stackebrandt and Ebers [52] have suggested a revised cut-off value of $\approx 99 \% 16 \mathrm{~S}$ rRNA identity for species delineation. We found that even using this stricter cut-off, we were not able to find evidence for delineating the type strains of A. calcoaceticus and A. pittii (99.3\%), and the type strain of A. pittii from A. nosocomialis strains NCTC 8102 and RUH2624 (99.5\%). Furthermore, when a phylogenetic tree is constructed from $16 \mathrm{~S}$ rRNA sequence data, the monophyly of the ACB complex was not preserved and the confidence values for most branches fall below $70 \%$ (Figure 1). Similar problems with using 16S rRNA gene sequences to resolve species have been reported in other genera [11,21].

Given the highly conserved nature of the $16 \mathrm{~S}$ rRNA gene sequences, we attempted to reconstruct a phylogeny based on more comprehensive gene set - the core genome of the genus. We found 911 orthologous coding sequences (CDSs) present in all thirty-eight strains, representing around a quarter of the average number of CDSs per strain. However, concerned that naïve use of this dataset might lead to problems due to homologous recombination, we selected a subset of 127 single-copy CDSs that showed with no signs of recombination according to three different measures (see Methods). These were concatenated, aligned and used to derive a phylogenomic tree (Figure 2). Interestingly, a tree constructed with no recombination filtering was nearly identical to the tree based on recombination-free CDSs (see Additional file 2).

This core genome tree generally supports the monophyletic status of the named species within the genus, with three exceptions: A. baumannii NCTC 7422 belongs in a deep-branching lineage with the $A$. parvus type strain DSM 16617, A. nosocomialis NCTC 10304 


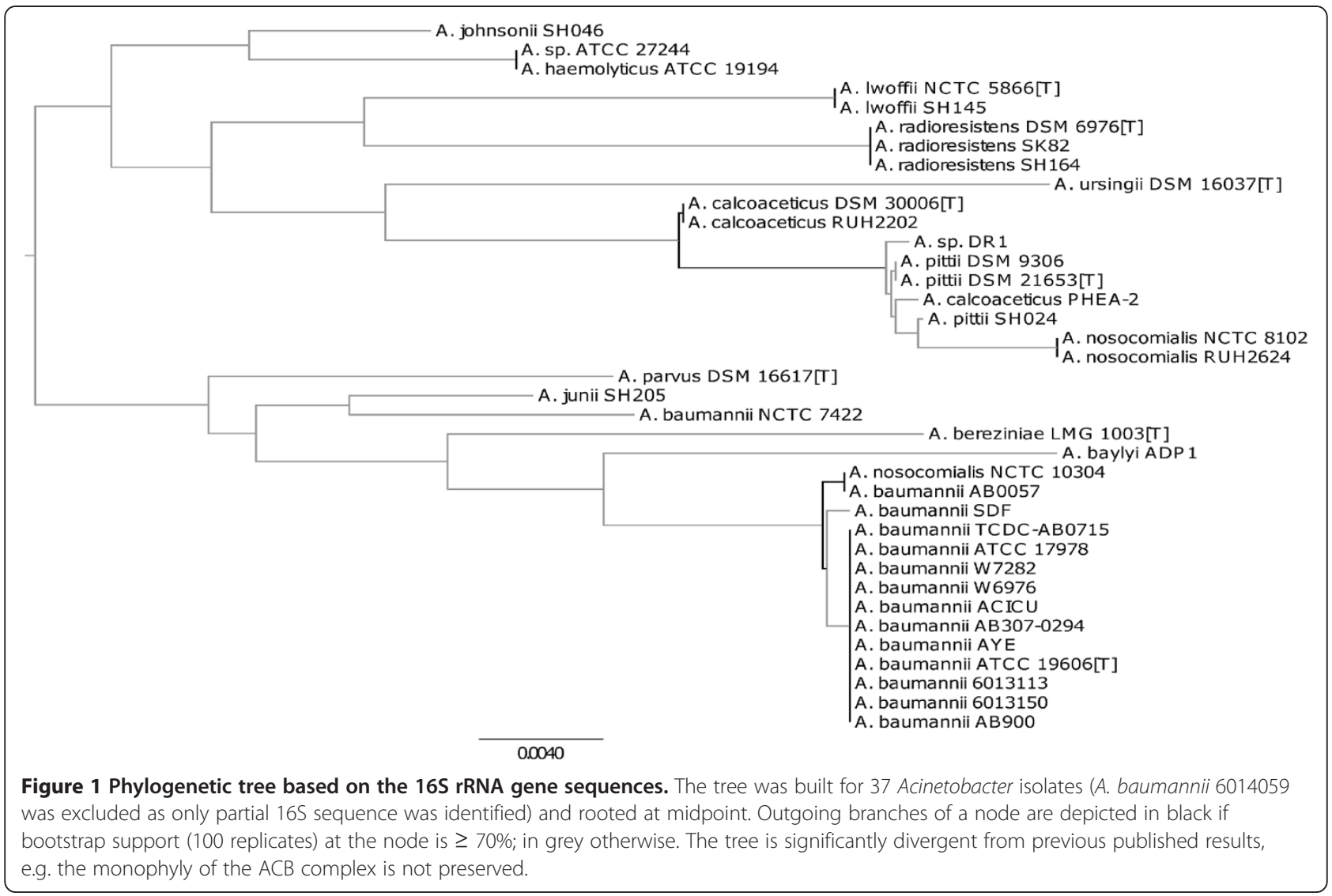

clusters within A. baumannii and A. calcoaceticus PHEA2 is closer to the three $A$. pittii strains than to the other two A. calcoaceticus strains. The first two strains have been genome-sequenced as part of this study and our results suggest they have been misclassified in the culture collection. PHEA-2 is an isolate from industrial wastewater that was genome-sequenced by $\mathrm{Xu}$ et al. [53]. Our core genome tree and comparisons of $16 \mathrm{~S}$ rRNA gene sequences show PHEA-2 to be closer to the three $A$. pittii strains than to the other two $A$. calcoaceticus strains, suggesting it too has been misclassified. Interestingly, the previously unclassified strain DR1 sits closest to the two $A$. calcoaceticus strains, while ATCC 27244 is closest to the species $A$. haemolyticus.

Once such reclassifications are taken into account, our core genome phylogenetic tree is consistent with the currently accepted genus taxonomy and also supports the monophyly of the ACB complex and of each of its four constituent species. Within A. baumannii, two lineages, international clones I and II, previously identified by comparative cell envelope protein profiling, ribotyping and AFLP genomic fingerprinting [53] are present as monophyletic groups in our tree. The tree obtained from the core genome is similar to a tree obtained from a recently described approach based on 42 ribosomal genes [15] (see Additional file 3).

\section{Rapid genomic approaches to species delineation}

Phylogenetic approaches are processor-intensive. We therefore evaluated genetic relatedness among the 38 strains using three rapid distance-based oligonucleotide and gene content approaches that avoid time-consuming calculations: the previously mentioned ANI, as well as K-string [54] and genome fluidity [55] approaches.

ANI relies on the identification of alignable stretches of nucleotide sequence in genome pairs, followed by a scoring and averaging of sequence identity, ignoring any divergent regions. The topology of the dendogram based on ANI analysis (Figure 3) is congruent with our core genome phylogenetic tree, confirming the misclassifications and new relationships already identified, while also showing the two international clones as separate lineages within $A$. baumannii.

The K-string composition approach [54] is based on oligopeptide content analysis of predicted proteomes. The divergence dendogram for $K=5$ (see Additional file 4) generally agrees with the results from the phylogenetic tree and ANI dendogram at species level. However, the major problem is that the K-string approach places A. baumannii SDF outside the ACB complex, probably reflecting the considerable difference in gene repertoires between this drug-sensitive strain and all other genome-sequenced $A$. baumannii strains. 

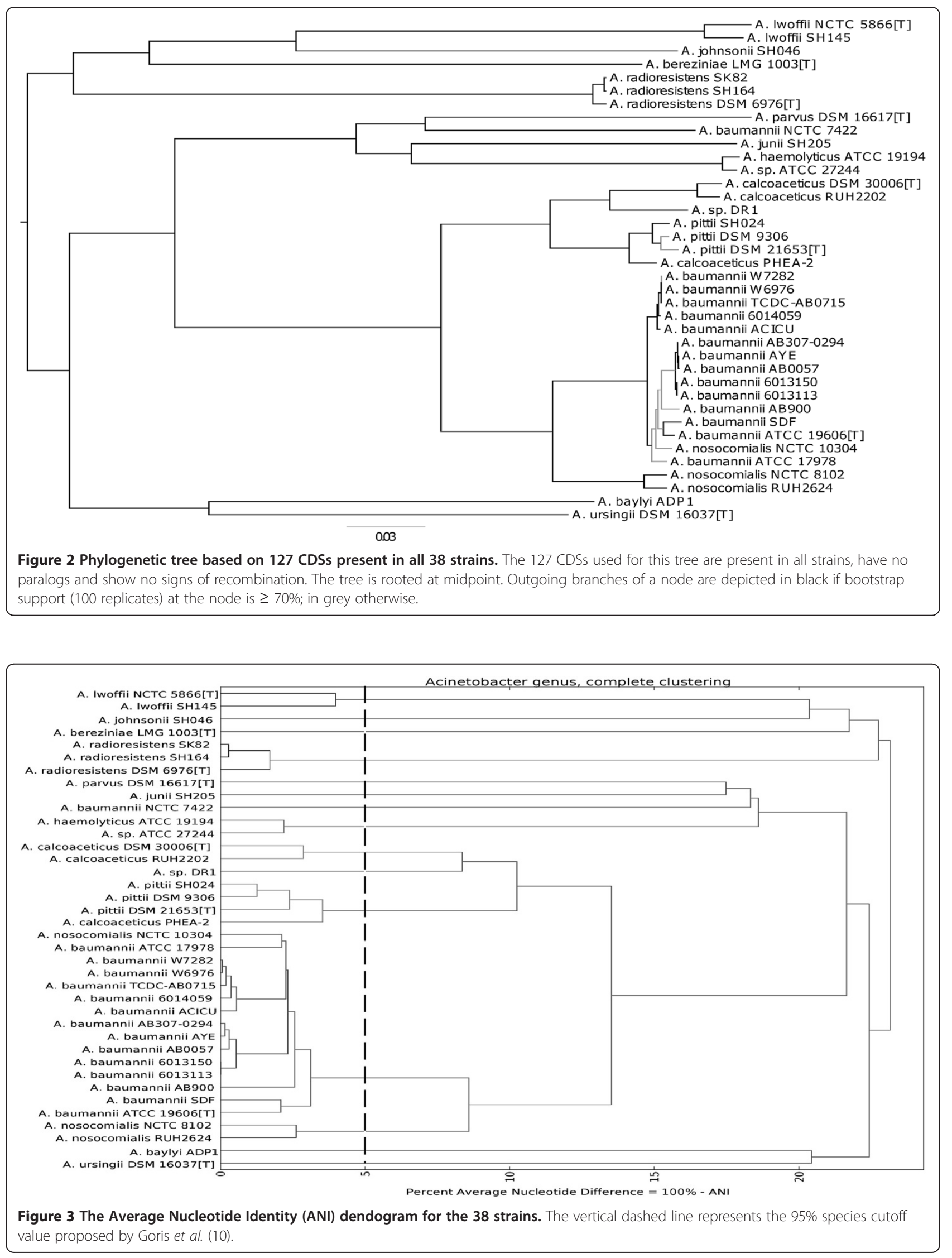
Genome fluidity provides a measure of the dissimilarity of genomes evaluated at the gene level [55]. A dendogram based on genomic fluidity (see Additional file 5) significantly differs from the results obtained with other techniques: $A$. baumannii SDF again sits outside the ACB complex, A. nosocomialis strains NCTC 8102 and RUH2624 now sit within the A. baumannii clade and PHEA-2 sits not with the $A$. pittii strains but with DR1 and the other A. calcoaceticus strains. We also performed pair-wise comparison of the gene content of the 38 strains, calculating the amount of the CDSs shared by each pair of strains (see Additional file 6). While strains from the same species generally share at least $80 \%$ of their CDSs, we found strains from different species exhibiting similar ratios. For example, A. calcoaceticus RUH2202 shares more than $80 \%$ of its CDS repertoire with DR1 and various A. nosocomialis, A. baumannii, A. pittii strains; PHEA-2 and DR1 share $88.1 \%$ of their CDSs. Based on gene content only, A. baumannii SDF is distinct from all other $A$. baumannii strains in our study (sharing at most $71.6 \%$ of its CDSs), which explains its placement in the K-string and genomic fluidity dendograms (see Additional files 4 and 5, respectively). These results indicate a potentially significant level of horizontal gene transfer among Acinetobacter species and illustrate an inability to delineate species based on gene content comparison only.

These findings suggest that ANI analyses provide results that are compatible with traditional and phylogenetic classifications, whereas K-string and genome fluidity approaches appear to be too strongly influenced by the effects of horizontal gene transfer to be consistent with previously accepted approaches.

\section{Defining species in Acinetobacter on the basis of whole- genome analyses}

The congruence of the phylogenetic tree and ANI dendogram with each other and with existing species definitions provides confidence that these techniques are fit for purpose in delineating species in the absence of phenotypic data. Furthermore, as Goris et al. suggest, the ANI approach provides a handy numerical cut-off at $95 \%$ identity to demarcate species boundaries, which corresponds to the $70 \% \mathrm{DDH}$ value [10]. When we applied this cut-off to our dataset, we were able to classify 37 of the strains into thirteen previously named species.

In line with the likely misclassification of strains, we observed that $A$. nosocomialis NCTC 10304 shares phylogenetic history and exhibits pair-wise ANI values greater than $95 \%$ with all 14 sequenced $A$. baumannii strains, thus confirming it should be designated A. baumannii NCTC 10304. Similar arguments apply for A. calcoaceticus PHEA-2 (new designation $A$. pittii PHEA-2) and $A$. sp. ATCC 27244 (A. haemolyticus ATCC 27244). However, the strain NCTC 7422 appears to be distinctive enough to represent new species. While the traditional polyphasic approach to taxonomy demands additional phenotypic characterization before these species can be named, on the basis of the analyses presented here, we propose the species name Acinetobacter bruijnii sp. nov. (N. L. gen. masc. n. bruijnii, of Bruijnius, named after Nicolaas Govert de Bruijn, Dutch mathematician) for strain NCTC 7422 and all future strains that are monophyletic and show $\geq 95 \%$ ANI to this strain.

It is interesting to note that our results based on core genome and ANI analyses differ from those based on AFLP patterns [56]; notably in the latter A. haemolyticus and $A$. junii do not cluster together nor does the cluster form a sister branch to the ACB complex; also A. johnsonii does not appear on the same deep-branch as A. lwoffii. This observation suggests that although AFLP is adept at species resolution, it appears to be unsuitable for phylogenetic analysis.

Several recent studies report alternative genomic approaches to bacterial taxonomy and species identification. These include in silico multilocus sequence analysis (MLSA), average amino acid identity (AAI) and ribosomal multilocus sequence typing (rMLST), which have been used to delineate species in the genera Neisseria, Vibrio and Mycoplasma [17,18,57]. Although MLSA can be used to infer phylogeny, this approach suffers from arbitrariness in choice of in genes which varies from one taxon to the next. Our proposed approach, core-genome phylogeny, can be considered an extension of MLSA and rMLST. However, as it is based on all shared CDSs in a given genus, it makes use of all potentially informative sequence sites. ANI, like AAI, measures pair-wise similarities between genome sequences but provides better resolution of species and sub-species [58,59].

\section{Conclusions}

The aim of this study has been to determine, using the genus Acinetobacter as a test case, whether genome sequence data alone are sufficient for the delineation and even definition of bacterial species. To this end, we explored the applicability of two broad approaches: sequence-based phylogenies for single and multiple gene and distance-based methods that include gene content comparisons (K-string and genomic fluidity) and wholegenome sequence similarities (ANI). We have found that a phylogenetic analysis of the genus Acinetobacter based on 16S rRNA gene sequences provides unreliable and uninformative results. By contrast, a core genome phylogenetic tree provides robust, informative results that are backwards compatible with the existing taxonomy.

Among the distance metrics, we found that approaches using gene content (K-string and genomic fluidity) led to anomalous conclusions, e.g., placing the SDF strain 
outside of the A. baumannii cluster, presumably because they are affected by horizontal gene transfer. In contrast, the easy-to-compute ANI results are congruent with the core genome phylogeny and traditional approaches. Using the core genome phylogeny and ANI approach, we found three misclassifications, one of which represents new species. These findings illustrate the need to genomesequence all strains archived in culture collections, which is likely to become technically and economically feasible in the near future.

We believe a combination of core genome phylogenetic analysis and ANI provides a feasible method for bacterial species delineation, in which species are defined as monophyletic groups of isolates that exhibit at least 95\% pair-wise ANI to each other. This approach combines a theoretically rigorous approach (sequence phylogeny) with a pragmatic metric (ANI) that provides a numerical cut-off that is backwards compatible and has been shown to be applicable to a diverse group of bacteria $[10,60]$.

Our sequence-based approach has several desirable characteristics. Firstly, it is capable of resolving the inconsistency in classification of genomospecies. For example, our results confirm the recent assignment of genomospecies 3 and $13 \mathrm{TU}$ to Latin binomials $A$. pittii and $A$. nosocomialis, respectively. Secondly, it provides a scalable and uniform approach that works for both culturable and non-culturable species, solving the problem in classifying non-culturable organisms, in an era when whole-genome sequences of such organisms can be recovered relatively easily via metagenomics or single-cell genomics. Thirdly, our approach is faster and cheaper than traditional taxonomic methods, as well as being easily replicable and transferable among research institutions. Finally a method that combines phylogeny and pragmatism falls in line with Darwin's vision of classification, as stated in the conclusion of Origin of Species: "Our classification will come to be, as far as they can be so made, genealogies..." [2].

\section{Methods}

\section{Strain selection and growth conditions}

Details of Acinetobacter strains used in this study are listed in Additional file 1. Acinetobacter baumannii W6976 and W7282 were provided by Drs. Mike Hornsey and David Wareham at Barts and The London NHS Trust, whilst the remaining strains were obtained from the UK, German and Belgium culture collections. Sequenced isolates were cultured in Nutrient broth or Tryptic soy medium at $25^{\circ} \mathrm{C}$ or $30^{\circ} \mathrm{C}$. DNA was extracted from single colony cultures using Qiagen 100/G Genomic-tips and quantified using Quant-iT PicoGreen dsDNA kits (Invitrogen). DNA was stored at $4^{\circ} \mathrm{C}$.

\section{Genomic sequencing and annotation}

DNA from thirteen isolates was sequenced by 454 GS FLX pyrosequencing (Roche, Branford, CT, USA) according to the standard protocol for whole-genome shotgun sequencing, producing an average of $450 \mathrm{bp}$ fragment reads. Draft genomes were assembled from flowgram data using Newbler 2.5 (Roche). The resulting contigs were annotated using the automated annotation pipeline on the xBASE server [61]. The genome sequences of the thirteen newly sequenced strains have been deposited in GenBank as whole genome shotgun projects (Table 1).

\section{Ortholog computation}

We computed the set of all orthologs within the 38 strains in our study with OrthoMCL [62] which performs a bidirectional best hit search in the amino-acid space, followed by a subsequent clustering step (percentMatchCutoff $=70$, evalueCutoff $=1 \mathrm{e}-05, \mathrm{I}=1.5)$. Predicted are 7,334 clusters of orthologous groups (COGs) containing 124,870 coding sequences (CDSs), which represents $95.7 \%$ of all good-quality CDSs (length at least 50 codons of which less than $2 \%$ are stop codons).

\section{Core genome phylogenetic tree construction}

Using the orthologs data, we extracted the genus core genome, i.e. the set of COGs which are present in each of the 38 strains (911 COGs). We filtered this set to exclude COGs containing paralogs and obtained a set of 827 single-copy COGs. The nucleotide gene sequences of each single-copy COG were aligned using MUSCLE 3.8.31 [63] with default parameters and the alignments were trimmed for quality, leading and trailing blocks using GBlocks 0.91b [64] with default parameters. After excluding 8 COGs with trimmed length $<50 \mathrm{bp}$, we screened the remaining 819 COGs for possible evidence of recombination using the PHI [65], MaxChi [66] and Neighbour similarity score [67] tests implemented in PhiPack (www.maths.otago.ac.nz/ dbryant/software/ PhiPack.tar) using 1000 permutations, window size $=50$ bp and p-value $<0.05$. To facilitate a more robust phylogeny construction, we selected only the 127 recombination-free COGs for which none of the three tests found evidence of recombination. The trimmed alignments of the 127 COGs were concatenated and used to build the tree by the approximately maximum-likelihood FastTree 2 [68] with 100 bootstrap replicates (created using SEQBOOT program from the PHYLIP package [69]. The resulting tree was visualized using FigTree (http://tree.bio. ed.ac.uk/software/figtree) and rooted at the mid-point.

The trees based on the $16 \mathrm{~S}$, the 819 single-copy COGs (no recombination filtering) and the 42 ribosomal genes were built in the same manner - multiple alignment of the nucleotide sequences with MUSCLE, trimming with 
GBlocks, and constructing bootstrapped trees (100 replicates) with FastTree 2, rooting them at mid-point.

\section{Average nucleotide identity (ANI)}

The ANI analysis was based on whole-genome data using the method proposed by Goris et al.[10]. Briefly, for each genome pair, one of the genomes was chosen as a query and split into consecutive $500 \mathrm{bp}$ fragments. These were then used to interrogate the second genome, designated the reference, using BLASTn [70] $(\mathrm{X}=150, \mathrm{q}=-1 \mathrm{~F}=\mathrm{F})$. For each query, the hit with the highest bit-score was selected and if the alignment exhibited at least 70\% identity and over $70 \%$ of the query fragment length, the hit was retained for further evaluation. The ANI score was computed as the mean identity of the retained hits. Based on the pair-wise ANI values, we compiled a distance matrix to represent the ANI divergence (which is defined as $100 \%$ - ANI) between the strains and used it to compute the ANI divergence dendogram with the hierarchical clustering package hcluster 0.2.0 adopting the complete linkage algorithm (http://pypi.python.org/pypi/hcluster).

Gene repertoire comparison (K-string and genomic fluidity) K-string analysis was based on the method proposed by Qi et al. [54]; for each proteome, its composition vector was computed by extracting the frequency of overlapping amino acid strings of length $\mathrm{K}$ and filtering out the random mutation background using a Markov model. The divergence between two genomes was computed by calculating the cosine function of the angle between the pair's composition vectors. The dendogram based on the pair-wise K-string distances was built as for ANI. The pair-wise genomic fluidity for each pair of genomes was computed using the ortholog data as suggested by Kislyuk et al. [55]. The dendogram was built as for ANI and $\mathrm{K}$-string.

\section{Additional files}

Additional file 1: The 38 sequenced Acinetobacter strains used in this study.

Additional file 2: Phylogenetic tree based on 819 core CDSs (without recombination filtering).

Additional file 3: Phylogenetic tree based on 42 ribosomal genes (Jolley et al. ) [15].

Additional file 4: K-string analysis of the 38 Acinetobacter strains used in this study.

Additional file 5: Genomic fluidity analysis of the 38 Acinetobacter strains used in this study.

Additional file 6: Pair-wise gene content comparison of the 38 Acinetobacter strains used in this study.

\section{Authors' contributions}

$\mathrm{JC}$ and $\mathrm{MH}$ designed and performed the study, analyzed data, drafted and revised the manuscript. NL analyzed data and revised the manuscript. CC performed the whole-genome sequencing and revised the manuscript.
MP conceived and designed the study and revised the manuscript. All authors read and approved the final manuscript.

\section{Acknowledgements}

We thank Dr. Mike Hornsey and Dr. David Wareham for the kind gift of isolates A. baumannii W6976 and W7282. JZ-MC, MRH, CC and MJP were supported by Medical Research Council grant G0901717; CC was also supported by the NIHR Surgical Reconstruction and Microbiology Research Centre; MRH and NJL were supported by Biotechnology and Biological Sciences Research Council grant BBE0111791.

Received: 31 July 2012 Accepted: 18 December 2012

Published: 23 December 2012

\section{References}

1. Linnaeus C: Systema naturæ, sive regna tria naturæ systematice proposita per classes, ordines, genera, \& species. Leiden: Apud Theodorum Haak; 1735.

2. Darwin C: On the origin of species by means of natural selection, or the preservation of favoured races in the struggle for life. London: John Murray, Albemarle Street; 1859.

3. Godreuil S, Cohan F, Shah H, Tibayrenc M: Which species concept for pathogenic bacteria?: An E-Debate. Infect Genet Evol 2005, 5:375-387.

4. Konstantinidis KT, Ramette A, Tiedje JM: The bacterial species definition in the genomic era. Philos Trans R Soc Lond B Biol Sci 2006, 361:1929-1940.

5. Van Belkum A, Tassios PT, Dijkshoorn L, Haeggman S, Cookson B, Fry NK, Fussing V, Green J, Feil E, Gerner-Smidt P, Brisse S, Struelens M, for the European Society of Clinical M, Infectious Diseases Study Group on Epidemiological M: Guidelines for the validation and application of typing methods for use in bacterial epidemiology. Clin Microbiol Infect 2007, 13:1-46.

6. Sneath PHA, Sokal RR: Numerical taxonomy: The principles and practice of numerical classification. San Francisco: W. H. Freeman; 1973.

7. Lee $K Y$, Wahl R, Barbu E: Contenu en bases purique et pyrimidiques des acides deoxyribonucleiques des bacteries. Ann Inst Pasteur 1956, 91:212-224.

8. Wayne LG, Brenner DJ, Colwell RR, Grimont PAD, Kandler O, Krichevsky ML, Moore LH, Moore WEC, Murray RGE, Stackebrandt E, Starr MP, Truper HG: Report of the ad hoc committee on reconciliation of approaches to bacterial systematics. Int J Syst Evol Microbiol 1987, 37:463-464.

9. Gevers D, Cohan FM, Lawrence JG, Spratt BG, Coenye T, Feil EJ, Stackebrandt E, Van de Peer Y, Vandamme P, Thompson FL, Swings J: Opinion: Re-evaluating prokaryotic species. Nat Rev Microbiol 2005, 3:733-739.

10. Goris J, Konstantinidis KT, Klappenbach JA, Coenye T, Vandamme P, Tiedje JM: DNA-DNA hybridization values and their relationship to wholegenome sequence similarities. Int J Syst Evol Microbiol 2007, 57:81-91.

11. Rosselló-Mora R, Amann R: The species concept for prokaryotes. FEMS Microbiol Rev 2001, 25:39-67.

12. Rappé MS, Giovannoni SJ: The uncultured microbial majority. Annu Rev Microbiol 2003, 57:369-394.

13. Eisen JA, Fraser CM: Phylogenomics: intersection of evolution and genomics. Science 2003, 300:1706-1707.

14. Konstantinidis KT, Tiedje JM: Prokaryotic taxonomy and phylogeny in the genomic era: advancements and challenges ahead. Curr Opin Microbiol 2007, 10:504-509.

15. Jolley KA, Bliss CM, Bennet JS, Bratcher HB, Brehoney CM, Colles FM, Wimalarathna HM, Harrison OB, Sheppard SK, Cody AJ, Maiden MCJ: Ribosomal multi-locus sequence typing: universal characterisation of bacteria from domain to strain. Microbiology 2012, 158:1005-1015.

16. Coenye T, Gevers D, de Peer $\mathrm{Y}$, Vandamme P, Swings J: Towards a prokaryotic genomic taxonomy. FEMS Microbiol Rev 2005, 29:147-167.

17. Thompson C, Vicente A, Souza R, Vasconcelos A, Vesth T, Alves N, Ussery D, lida T, Thompson F: Genomic taxonomy of Vibrios. BMC Evol Biol 2009, 9:258.

18. Thompson CC, Vieira NM, Vicente ACP, Thompson FL: Towards a genome based taxonomy of Mycoplasmas. Infect Genet Evol 2011, 11:1798-1804.

19. Ibrahim A, Gerner-Smidt P, Liesack W: Phylogenetic relationship of the twenty-One DNA groups of the genus Acinetobacter as revealed by 16S ribosomal DNA sequence analysis. Int J Syst Evol Microbiol 1997, 47:837-841 
20. Janda JM, Abbott SL: 16S RRNA gene sequencing for bacterial identification in the diagnostic laboratory: pluses, perils, and pitfalls. J Clin Microbiol 2007, 45:2761-2764.

21. Fox GE, Wisotzkey JD, Jurtshuk P: How close is close: $16 \mathrm{~S}$ rRNA sequence identity may not be sufficient to guarantee species identity. Int J Syst Evol Microbiol 1992, 42:166-170

22. Brisou J, Prevot AR: Etudes de systematique bacterienne. X. Revision des especes reunies dans le genre Achromobacter. Ann Inst Pasteur 1954, 86:722-728

23. Baumann P, Doudoroff M, Stanier RY: A study of the Moraxella group II. Oxidative-negative species (genus Acinetobacter). J Bacteriol 1968, 95:1520-1541.

24. Peleg AY, Seifert $H$, Paterson DL: Acinetobacter baumannii: Emergence of a Successful Pathogen. Clin Microbiol Rev 2008, 21:538-582.

25. Lorenz MG, Reipschlager K, Wackernagel W: Plasmid transformation of naturally competent Acinetobacter calcoaceticus in non-sterile soil extract and groundwater. Arch Microbiol 1992, 157:355-360.

26. Poirel L, Figueiredo S, Cattoir V, Carattoli A, Nordmann P: Acinetobacter radioresistens as a silent source of carbapenem resistance for Acinetobacter spp. Antimicrob Agents Chemother 2008, 52:1252-1256.

27. Vaneechoutte M, Young DM, Ornston LN, De Baere T, Nemec A, Van Der Reijden T, Carr E, Tjernberg I, Dijkshoorn L: Naturally transformable Acinetobacter sp. strain ADP1 belongs to the newly described species Acinetobacter baylyi. Appl Environ Microbiol 2006, 72:932-936.

28. Baumann P: Isolation of Acinetobacter from soil and water. J Bacteriol 1968, 96:39-42.

29. Jung J, Park W, Baek JH: Complete genome sequence of the dieseldegrading Acinetobacter sp. strain DR1. J Bacteriol 2010, 192:4794-4795.

30. Zhan Y, Yu H, Yan Y, Chen M, Lu W, Li S, Peng Z, Zhang W, Ping S, Wang J, Lin M: Genes involved in the benzoate catabolic pathway in Acinetobacter calcoaceticus PHEA-2. Curr Microbiol 2008, 57:609-614.

31. Park YS, Lee H, Lee KS, Hwang SS, Cho YK, Kim HY, Uh Y, Chin BS, Han SH, Jeong SH, Lee K, Kim JM: Extensively drug-resistant Acinetobacter baumannii: risk factors for acquisition and prevalent OXA-type carbapenemases-a multicentre study. Int J Antimicrob Ag 2010, 36:430-435.

32. Grosso F, Quinteira S, Peixe L: Emergence of an extreme-drug-resistant (XDR) Acinetobacter baumannii carrying blaOXA-23 in a patient with acute necrohaemorrhagic pancreatitis. J Hosp Infect 2010, 75:82-83.

33. Turton JF, Shah J, Ozongwu C, Pike R: Incidence of Acinetobacter species other than A. baumannii among clinical isolates of Acinetobacter: Evidence for emerging species. J Clin Microbiol 2010, 48:1445-1449.

34. Gerner-Smidt P, Tjernberg I, Ursing J: Reliability of phenotypic tests for identification of Acinetobacter species. J Clin Microbiol 1991, 29:277-282.

35. Janssen P, Maquelin K, Coopman R, Tjernberg I, Bouvet P, Kersters K, Dijkshoorn L: Discrimination of Acinetobacter Genomic Species by AFLP Fingerprinting. Int J Syst Bacteriol 1997, 47:1179-1187.

36. Janssen P, Coopman R, Huys G, Swings J, Bleeker M, Vos P, Zabeau M, Kersters K: Evaluation of the DNA fingerprinting method AFLP as a new tool in bacterial taxonomy. Microbiology 1996, 142:1881-1893.

37. Dijkshoorn L, van Harsselaar B, Tjernberg I, Bouvet PJM, Vaneechoutte M: Evaluation of Amplified Ribosomal DNA Restriction Analysis for Identification of Acinetobacter Genomic Species. Syst Appl Microbiol 1998, 21:33-39.

38. Vaneechoutte M, Dijkshoorn L, Tjernberg I, Elaichouni A, de Vos P, Claeys G, Verschraegen G: Identification of Acinetobacter genomic species by amplified ribosomal DNA restriction analysis. J Clin Microbiol 1995, 33:11-15.

39. Nemec A, Krizova L, Maixnerova M, van der Reijden TJK, Deschaght P, Passet V, Vaneechoutte M, Brisse S, Dijkshoorn L: Genotypic and phenotypic characterization of the Acinetobacter calcoaceticus-Acinetobacter baumannii complex with the proposal of Acinetobacter pittii sp. nov. (formerly Acinetobacter genomic species 3 ) and Acinetobacter nosocomialis sp. nov. (formerly Acinetobacter genomic species 13TU). Res Microbiol 2011, 162:393-404.

40. Nemec A, De Baere T, Tjernberg I, Vaneechoutte M, van der Reijden TJ, Dijkshoorn L: Acinetobacter ursingii sp. nov. and Acinetobacter schindleri sp. nov., isolated from human clinical specimens. Int J Syst Evol Microbiol 2001, 51:1891-1899.

41. Bonnin RA, Poirel L, Nordmann P: AbaR-type transposon structures in Acinetobacter baumannii. J Antimicrob Chemother 2012, 67:234-236.
42. Bouvet PJM, Grimont PAD: Taxonomy of the Genus Acinetobacter with the Recognition of Acinetobacter baumannii sp. nov., Acinetobacter haemolyticus sp. nov., Acinetobacter johnsonii sp. nov., and Acinetobacter junii sp. nov. and Emended Descriptions of Acinetobacter calcoaceticus and Acinetobacter Iwoffii. Int J Syst Evol Microbiol 1986, 36:228-240.

43. Kim Y-O, Kim W-J, Choi S-H, Kim D-S, Kim D-W, Lee J-S, Kong HJ, Nam B-H, Kim B-S, Lee S-J, Park H-S, Chae S-H: Genome Sequence of Acinetobacter sp. Strain P8-3-8, Isolated from Fistularia commersonii in Vietnam. J Bacteriol 2011, 193:4288-4289.

44. Heuer H, Kopmann C, Binh CTT, Top EM, Smalla K: Spreading antibiotic resistance through spread manure: characteristics of a novel plasmid type with low \%G+C content. Environ Microbiol 2009, 11:937-949.

45. Nemec A, Dijkshoorn L, Cleenwerck I, De Baere T, Janssens D, van der Reijden TJK, Jezek P, Vaneechoutte M: Acinetobacter parvus sp. nov., a small-colony-forming species isolated from human clinical specimens. Int J Syst Evol Microbiol 2003, 53:1563-1567.

46. Lima-Mendez G, Van Helden J, Toussaint A, Leplae R: Prophinder: a computational tool for prophage prediction in prokaryotic genomes. Bioinformatics 2008, 24:863-865.

47. Nemec A, Musílek M, Šedo O, De Baere T, Maixnerová M, van der Reijden TJK, Zdráhal Z, Vaneechoutte M, Dijkshoorn L: Acinetobacter bereziniae sp. nov. and Acinetobacter guillouiae sp. nov., to accommodate Acinetobacter genomic species 10 and 11, respectively. Int J Syst Evol Microbiol 2010, 60:896-903.

48. Nishimura $\mathrm{Y}$, Ino T, lizuka $\mathrm{H}$ : Acinetobacter radioresistens sp. nov. Isolated from Cotton and Soil. Int J Syst Evol Microbiol 1988, 38:209-211.

49. Pessione E, Giunta C: Acinetobacter radioresistens metabolizing aromatic compounds. 2. Biochemical and microbiological characterization of the strain. Microbios 1997, 89:105-117.

50. Stackebrandt E, Goebel BM: Taxonomic note: A place for DNA-DNA reassociation and $16 \mathrm{~S}$ rRNA sequence analysis in the present species definition in bacteriology. Int I Syst Evol Microbiol 1994, 44:846-849.

51. Keswani J, Whitman WB: Relationship of $16 \mathrm{~S}$ rRNA sequence similarity to DNA hybridization in prokaryotes. Int J Syst Evol Microbiol 2001, 51:667-678

52. Stackebrandt E, Ebers J: Taxonomic parameters revisited: tarnished gold standards. Microbiol Today 2006, 33:152-155.

53. Xu Y, Chen M, Zhang W, Lin M: Genetic organization of genes encoding phenol hydroxylase, benzoate 1,2-dioxygenase alpha subunit and its regulatory proteins Acinetobacter calcoaceticus PHEA-2. Curr Microbiol 2003, 46:235-240

54. Dijkshoorn L, Aucken H, Gerner-Smidt P, Janssen P, Kaufmann ME, Garaizar J, Ursing J, Pitt TL: Comparison of outbreak and nonoutbreak Acinetobacter baumannii strains by genotypic and phenotypic methods. J Clin Microbiol 1996, 34:1519-1525.

55. Qi J, Wang B, Hao B-l: Whole proteome prokaryote phylogeny without sequence alignment: a K-string composition approach. J Mol Evol 2004, 58:1-11.

56. Kislyuk A, Haegeman B, Bergman N, Weitz J: Genomic fluidity: an integrative view of gene diversity within microbial populations. BMC Genomics 2011, 12:32

57. Janssen P, Maquelin K, Coopman R, Tjernberg I, Bouvet P, Kersters K, Dijkshoorn L: Discrimination of Acinetobacter Genomic Species by AFLP Fingerprinting. Int J Syst Evol Microbiol 1997, 47:1179-1187.

58. Bennett JS, Jolley KA, Earle SG, Corton C, Bentley SD, Parkhill J, Maiden MCJ: A genomic approach to bacterial taxonomy: an examination and proposed reclassification of species within the genus Neisseria. Microbiology 2012, 158:1570-1580.

59. Rosselló-Mora R: Updating Prokaryotic Taxonomy. J Bacteriol 2005, 187:6255-6257.

60. Konstantinidis KT, Tiedje JM: Towards a genome-based taxonomy for prokaryotes. J Bacteriol 2005, 187:6257-6264.

61. Richter M, Rosselló-Móra R: Shifting the genomic gold standard for the prokaryotic species definition. PNAS 2009, 106:19126-19131.

62. Chaudhuri RR, Loman NJ, Snyder LAS, Bailey CM, Stekel DJ, Pallen MJ: xBASE2: a comprehensive resource for comparative bacterial genomics. Nucleic Acids Res 2008, 36:D543-D546.

63. Li L, Stoeckert CJ Jr, Roos DS: OrthoMCL: identification of ortholog groups for eukaryotic genomes. Genome Res 2003, 13:2178-2189.

64. Edgar RC: MUSCLE: multiple sequence alignment with high accuracy and high throughput. Nucleic Acids Res 2004, 32:1792-1797. 
65. Talavera G, Castresana J: Improvement of phylogenies after removing divergent and ambiguously aligned blocks from protein sequence alignments. Syst Biol 2007, 56:564-577.

66. Bruen TC, Philippe $H$, Bryant D: A simple and robust statistical test for detecting the presence of recombination. Genetics 2006, 172:2665-2681.

67. Smith JM: Analyzing the mosaic structure of genes. J Mol Evol 1992, 34:126-129.

68. Jakobsen IB, Easteal S: A program for calculating and displaying compatibility matrices as an aid in determining reticulate evolution in molecular sequences. Comput Appl Biosci 1996, 12:291-295.

69. Price MN, Dehal PS, Arkin AP: FastTree: Computing Large Minimum Evolution Trees with Profiles instead of a Distance Matrix. Mol Biol Evol 2009, 26:1641-1650.

70. Felsenstein J: PHYLIP - Phylogeny Inference Package (Version 3.2). Cladistics 1989, 5:164-166.

71. Altschul SF, Madden TL, Schäffer AA, Zhang J, Zhang Z, Miller W, Lipman DJ: Gapped BLAST and PSI-BLAST: a new generation of protein database search programs. Nucleic Acids Res 1997, 25:3389-3402.

doi:10.1186/1471-2180-12-302

Cite this article as: Chan et al:: Defining bacterial species in the genomic era: insights from the genus Acinetobacter. BMC Microbiology 2012 12:302.

\section{Submit your next manuscript to BioMed Central and take full advantage of:}

- Convenient online submission

- Thorough peer review

- No space constraints or color figure charges

- Immediate publication on acceptance

- Inclusion in PubMed, CAS, Scopus and Google Scholar

- Research which is freely available for redistribution 